\title{
Problèmes diphasiques rencontrés dans les générateurs de vapeur des chaudières nucléaires PWR
}

\section{Comportement transitoire aux faibles charges}

\section{Two-phase problems in the steam generators of PWR boilers}

\author{
Transient low-pressure effects
}

\author{
Jean-Pierre Hennebicq *, Robert Larminaux * * Jean Tassart * \\ ${ }^{*}$ EDF - Direction des Études et Recherches, Chatou \\ ${ }^{*}$ EDF-SEPTEN, Direction de l'Équipement
}

\section{Introduction}

On décrira les problèmes rencontrés à l'occasion de la mise en œuvre des générateurs de vapeur ("GV") PWR à circulation naturelle de type Westinghouse et construits par Framatome pour les centrales d'EDF.

Au régime nominal, les deux tiers du volume secondaire de ces générateurs de vapeur sont parcourus par de l'émulsion. Un modèle numérique unifilaire EDF ${ }^{(1)}$ fournit les valeurs numériques de la figure 1. La dissymétrie de l'échange thermique se répercute sur l'écoulement secondaire.

On se limitera aux problèmes liés au fonctionnement normal et à ce qui relève strictement de l'écoulement diphasique. On insistera sur le problème de la régulation du niveau aux basses charges.

Diphasique et tenue du matériel en régime normal

Corrosion des tubes au voisinage de la plaque tubulaire

La corrosion des tubes est favorisée, dans les zones d'échange à flux élevé, par :

- l'assèchement et remouillage de la paroi,

- l'accumulation de boues dans les zones de faibles vitesses d'écoulement.

Or, la production de bulles en jambe chaude est importante dès le voisinage de la plaque tubulaire sur les GV de type Westinghouse mis en service avant 1975.

D'importants moyens ont étê mis en œuvre, notamment par Westinghouse, Framatome et EDF, pour mieux

(1) Modèle GVTRAP. connaître l'écoulement au pied du faisceau :

- mesures aux courants de Foucault sur des GV en exploitation (localisation des boues),

- modèle numérique diphasique homogène bidimensionnel de Westinghouse (approches de la configuration de l'écoulement) ${ }^{(2)}$

- instrumentation succincte sur GV déjà installés, dont un à Fessenheim 2 (étude de l'écoulement dans la redescente),

- maquettes tridimensionnelles en eau-air, de visualisation de l'écoulement, à $E D F$, avec vélocimètre à laser (influences de la troisième dimension et de la viscosité),

- instrumentation plus complète, par EDF, de deux GV bientôt en fonctionnement (mesure du taux de circulation, données sur l'écoulement au voisinage de la plaque tubulaire, pression au dôme du GV).

Les trois premiers moyens cités ont permis d'améliorer l'écoulement au bas des nouveaux $G V$, essentiellement par addition d'une plaque déflectrice horizontale à trou central, blocage de la "rue d'eau" (by-pass supprimé et écoulement plus centripète) et augmentation du "taux de circulation".

Des modèles numériques tridimensionnels, en diphasique homogène, sont développés aux USA et en Europe. De tels modèles semblent également nécessaires pour étudier l'écoulement dans la zone cintrée du faisceau.

Une maquette cylindrique en eau-vapeur respectant les conditions de simulitude, conservant des vitesses d'écoulement et un diamètre de faisceau suffisants, nécessiterait une puissance d'au moins $35 \mathrm{MW}$. Le fréon permettrait de ramener cette valeur à $4 \mathrm{MW}$.

(2) EDF a récemment mis au point un modèle comparable, "BIGEVE" (1) dont un résultat est donné en figure 2. 


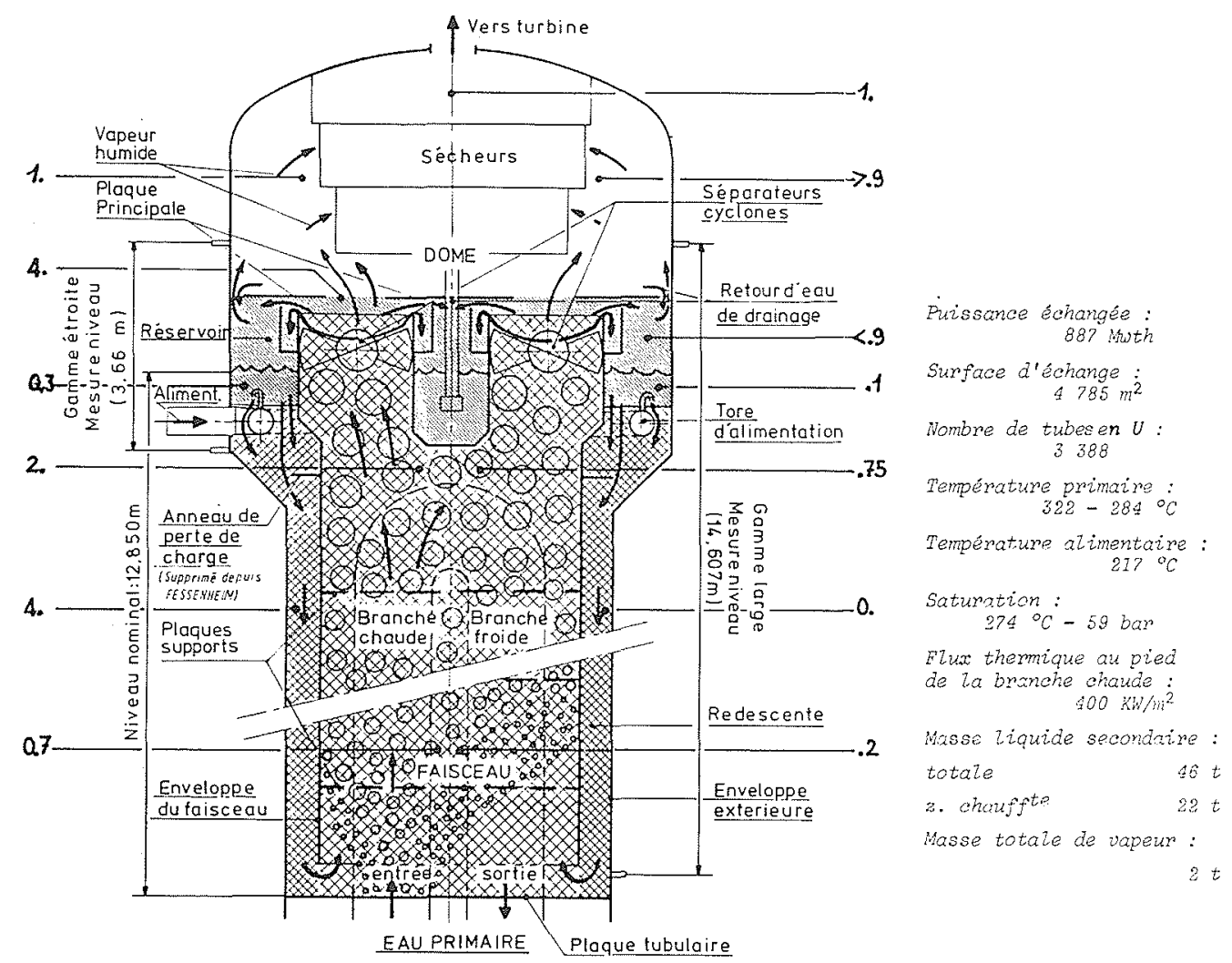

Figure 1 - Générateur de vapeur type 51A (Fessenheim) écoulement secondaire Vitesse secondaire moyenne $\mathrm{m} / \mathrm{s}$ Taux de vide moyen

L'instrumentation de GV comme celle de telles maquettes pose le problème des mesures en écoulements diphasiques.

\section{Striction à la traversée des plaques supports}

Le mauvais balayage des tubes à la traversée des plaques-supports en acier au carbone à perçage circulaire peut, par corrosion de ces plaques, entraîner la striction puis la fissuration des tubes.

Le phénomène a été reproduit en maquettes. Néanmoins, la thermohydraulique à la traversée des plaquessupports est mal connue : les rares résultats expérimentaux ne concordent pas quant au risque d'assèchement.

L'adoption d'un dessin quadrifolié des traversées devrait contribuer à l'amélioration de la fiabilité. D'autres conceptions semblent pouvoir donner également satisfaction : types Combustion Engineering et KWU (BREDA).

\section{Risques d'instabilité hydroélastique du faisceau}

L'interaction fluide-faisceau est déterminante au niveau des cintres où le taux de vide dépasse $75 \%$. Deux inconnues subsistent quant à l'évaluation des marges de stabilité : le champ des vitesses normales aux plus grands cintres, les marges résultant du glissement vapeur-liquide.

Des essais sur maquettes sont en cours sur ces deux points, tandis que le soudage de jauges de déformation à l'intérieur de cintres d'un GV de type $51 \mathrm{M}$ permettra de mesurer les sollicitations réellles des tubes.

\section{Diphasique et performances}

\section{Pression de vapeur}

L'incertitude avec laquelle on connaît tant l'écart réel de température primaire-secondaire que la conductivité des tubes ne permet ni de critiquer la corrélation d'échange de Jens et Lotte - Westinghouse en ébullition nuclée, ni de mettre en évidence l'existence d'éventuelles zones d'assèchement de quelques pour cent de la surface d'échange.

Sur les GV des chaudières de 1300 MWe commandées par EDF, la sous-saturation à l'admission en jambe chaude est accrue en y envoyant $80 \%$ du débit alimentaire. On réduit ainsi le risque de corrosion mais on diminue l'écart de température primaire secondaire en jambe froide, ce qui tend à abaisser la pression secondaire.

Inversement, l'intérêt de la création d'un économiseur en jambe froide dépend notamment de sa répercussion sur les conditions d'échange et de corrosion au pied des deux branches du faisceau. Ainsi, une amélioration de la connaissance de ces dernières permettrait une meilleure évaluation du compromis optimal performances-fiabilité.

L'expérience des GV à économiseur (notamment de Westinghouse), ainsi que des essais menés sur maquettes 
en eau-vapeur, pourraient apporter des éclaircissements sur ces problèmes.

\section{Séchage de la vapeur}

L'humidité de la vapeur à la sortie des GV est mesurée à $20 \%$ près en relatif par traceur radioactif injecté dans l'eau alimentaire.

L'expérience des GV en fonctionnement et des essais sur maquettes a montré le rôle primordial joué par la zone intermédiaire cyclones-sécheurs :

- préconisée par Westinghouse, la réduction des orifices de sortie des séparateurs dans la plaque principale, accompagnée d'un accroissement de la section des espaces périphériques plaque-virole (fig. 1), s'est montrée efficace sur les $G V$ en exploitation ;

- la sensibilité des performances des sécheurs à la pres. sion dynamique de la vapeur incidente a conduit EDF à mettre au point, sur une maquette à eau de visualisation de l'espace intermédiaire, un déflecteur permettant une bonne homogénéisation des vitesses à l'entrée des sécheurs ;

- sur maquettes eau-fréon, EDF a montré l'efficacité d'un dispositif de drainage de la plaque principale des GV, réduisant l'humidité à l'amont des sécheurs par réduction du réentraînement.

Ces améliorations, avec quelques perfectionnements dus à Framatome, devraient assurer une bonne qualité de la vapeur à la sortie des GV des chaudières de 900 MWe d'EDF.

\section{Fonctionnement transitoire normal}

\section{Stabilité hydrodynamique de la boucle de circulation}

La stabilité hydrodynamique des GV de type Westinghouse du palier EDF 900 MWe s'est montrée suffisante lors des essais.

Les périodes propres et amortissements de la boucle avaient été évalués par Framatome (modèle numérique par fonction de transfert linéaire) et EDF (représentation transitoire par différences finies ${ }^{(3)}$ ). Les calculs EDF ont montré la sensibilité de l'amortissement aux conditions aux limites côté vapeur.

Les limites de stabilité des GV du palier $1300 \mathrm{MWe}$ ne semblent pas très éloignées du régime nominal, or ni la répartition des pertes de charge ni le taux de circulation ne sont assez bien connus pour rendre les modèles de calculs suffisamment crédibles.

Un essai spécifique à la charge nominale d'une tranche de Bugey n'a pas permis d'évaluer l'amortissement des oscillations de très faible amplitude obtenues indépendamment de toute régulation. Des essais sur une maquette axiale chauffante au fréon permettront une étude détaillée du problème. Le dépouillement en sera fait à l'aide d'un modèle de calcul EDF à fonction de transfert linéarisée ${ }^{(4)}$.

(3) Modèles GVMATIC (2) et GVTRAP.

(4) Modèle EOLE.

\section{Mesure du niveau}

La mesure du niveau GV en "gamme étroite" (fig. 1) remplit une fonction de régulation et une fonction de protection.

Le "niveau" est défini à partir de la différence de pressions statiques entre deux prises situées sur une même génératrice de la virole, l'une en phase liquide et l'autre en phase vapeur. Il représente approximativement la masse d'eau liquide située en zone périphérique du $G V$, entre deux plans horizontaux passant par les deux prises.

Cette mesure peut être perturbée, de façon transitoire ou permanente, par les conditions de l'écoulement secondaire entre les deux prises : pertes de charge parasites, pressions dynamiques, hétérogénéités de l'écoulement liquide... Mais EDF se pose surtout la question de la représentativité d'une telle mesure lors d'une dépressurisation accidentelle.

Des développements sont en cours sur de nouveaux dispositifs de mesure : thermocouples chauffants, mesure de conductivité, capteurs acoustiques, ...

\section{Régulation du niveau aux basses charges}

La régulation des niveaux des générateurs de vapeur de type Westinghouse, manuelle en-dessous de $15 \%$ de charge jusqu'en fin 1975 , constituait la principale difficulté de conduite et l'une des principales causes d'indisponibilité en périodes de démarrage. En 1973, EDF entreprit l'analyse de ce problème à l'aide d'un modèle numérique mis au point à cet effet. A l'occasion des études menées pour Tihange, EDF put bénéficier de l'expérience des démarrages de Doel 1 et de Tihange 1 aux côtés des équipes belges de ACEC, des EBES ${ }^{(5)}$ et de Laborelec.

La représentation "ponctuelle" du GV alors utilisée dans les modèles d'étude du fonctionnement général ne rendait pas compte de telles difficultés. Aussi, EDF mit au point un modèle diphasique avec glissement de représentation axiale d'un GV découpant la boucle de circulation en un grand nombre de tranches ("CrVMATIC" : (2)). Un modèle analogique représentant deux GV en parallèle a permis d'évaluer l'importance réelle du couplage entre plusieurs GV (3).

Analyse du problème à l'aide de modèles de calcul

Le modèle numérique a permis de retrouver l'évolution exacte des phénomènes enregistrés à Chooz (fig. 3) à basse charge avec la chaîne de régulation initialement proposée par le constructeur (fig. 5).

Les réponses indicielles du modèle axial à des échelons de débit d'eau et de débit de vapeur (fig. 4 et 5) comportent deux temps successifs :

- redistribution rapide de la masse d'eau liquide dans le GV,

apparition, sur la mesure du niveau "gamme étroite", de la variation du bilan de masse global.

(5) ACEC : Ateliers de Constructions Electriques de Charleroi.

EBES: Sociétés réunies d'Energie du Bassin de l'Escaut. 


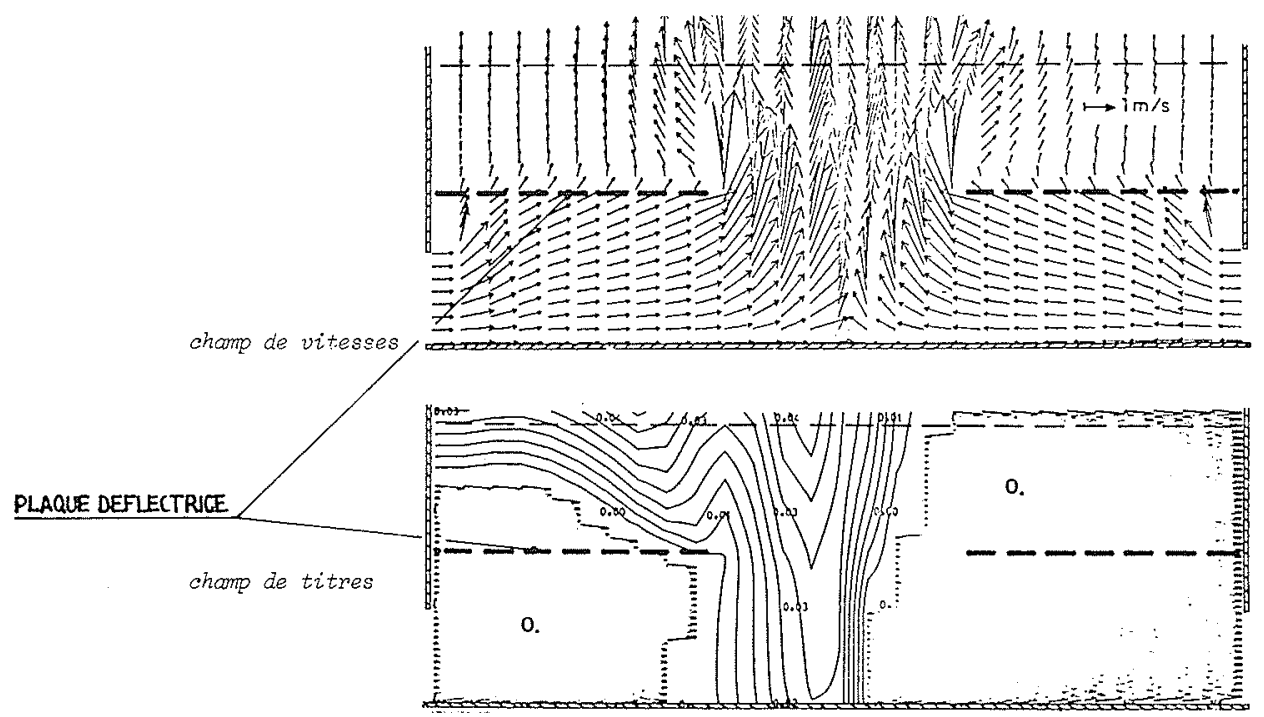

Figure 2 - Ecoulement au bas d'un générateur de vapeur 68-19 (Palier EDF-1300 Mwe) calcul bidimensionnel "BIGEVE", EDF

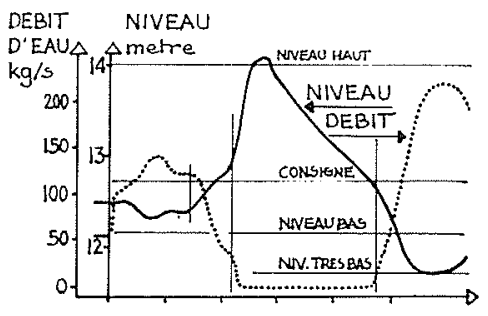

$$
\text { DEBIT DE VAPEUR }
$$

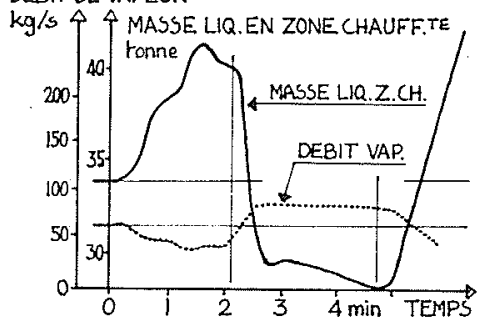

Figure 3 - Simulation d'un transitoire typique enregistré à Chooz.
Pour les régimes normaux de fonctionnement, on peut tracer la courbe de la masse d'eau dans le GV à niveau constant en fonction de la charge (fig. 6). Cette courbe permet de prévoir approximativement l'amplitude $\mathrm{du}$ gonflement et du tassement provoqués par un échelon de débit de vapeur.

La plus grande amplitude du tassement-gonflement aux basses charges est due à la non-linéarité de la fonction donnant le taux de vide en fonction du titre de vapeur.

Mise au point d'une nouvelle chaîne de régulation de niveau (4)

- Des essais à la centrale belge de Doel et la simulation analogique des GV couplés ont fait apparaître la nécessité de tenir compte du couplage vapeur dans la définition de la chaîne de régulation (3).

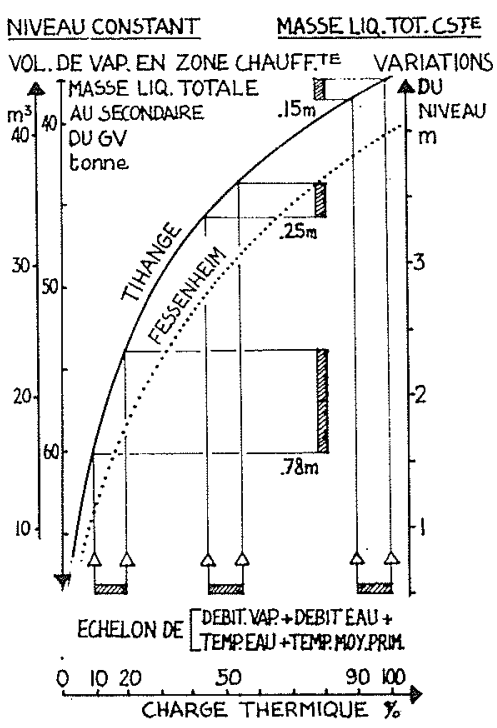

Figure 6 - Tassement - gonflement du niveau et volume de vapeur en zone chauffante.

Figures 4 èt 5 - Rép. indicielles du modèle num. EDF. 

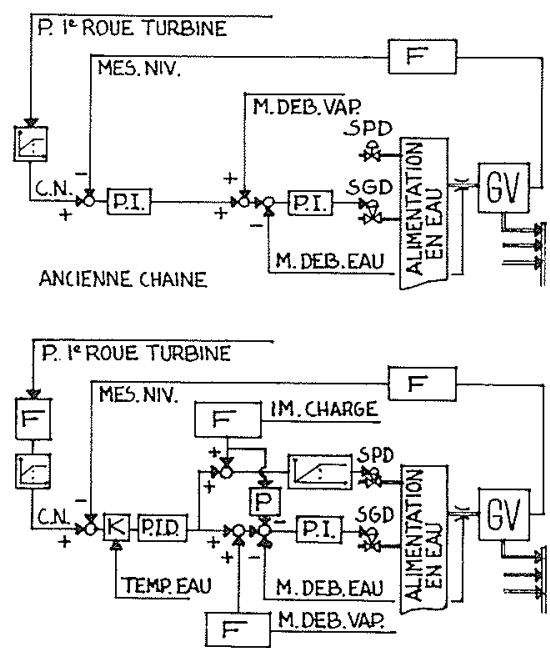

NOUVELLE CHAINE

Figure 7 - Adaptation aux basses charges de la chaine de régulation du niveau.

- Au-dessous de $15 \%$ de charge, les mesures de débits d'eau et de vapeur utilisées pour la régulation de la soupape grand débit sont inutilisables. Une chaine spécifique est donc nécessaire pour commander la soupape petit débit, tandis que le régulateur de niveau est commun aux deux chaînes (fig. 7).

- Une adaptation dynamique de l'action de tendance a permis de soulager la tâche du régulateur de niveau lors de variations de charge (fig. 6).

- L'étude de stabilité de la boucle fermée du niveau a permis de définir une loi de variation linéaire du gain proportionnel en fonction de la charge. La correspondance charge-température alimentaire en régime normal a permis de choisir cette dernière comme critère d'adaptation du gain.

- Aux charges élevées, la soupape petit débit est grande ouverte. A basse charge, la soupape grand débit est fermée : on ajoute une tension de polarisation au signal de débit d'eau de la chaine gros débit. Cette tension est annulée dès que le signal de tendance "petit débit" devient supérieur à une certaine valeur (Procédé automatique Framatome).

Cette régulation représente un progrès considérable par rapport à la conduite manuelle : en période d'exploitation normale, les opérateurs doivent se fier à la régulation. Dans un certain nombre de cas cependant, le faible gain du régulateur de niveau ne permet pas de rattraper les insuffisances du prépositionnement de la soupape "petit débit".

\section{Conclusion}

La schématisation unifilaire du fonctionnement transitoire des GV PWR à circulation naturelle a largement contribué à la solution, en voie d'achèvement, du problème de la régulation du niveau. Des données expérimentales manquent encore pour parfaire cette représentation.

La lutte contre les corrosions a demandé la mise en œuvre de nombreux moyens d'investigation. Elle a amené à augmenter le taux de circulation, nécessitant ainsi l'étude d'autres problèmes, qui ont été ou seront prochainement résolus.

Les besoins de la sûreté aussi bien que la recherche d'une optimisation économique poussent à développer des moyens d'études et d'essais supplémentaires : modèles numériques tridimensionnels à deux phases, instrumentation de mesure des vitesses, taux de vide et pertes de charges, enfin maquettes à eau-vapeur et à fréon. L'instrumentation fine de GV réels et l'analyse de leurs évolutions dynamiques semblent pouvoir être particulièrement profitables.

\section{Références}

[1]COEFFE, TODREAS. - Modèle mathématique bidimensionnel d'écoulement diphasique dans un générateur de vapeur. XVIII ${ }^{\mathrm{e}}$ Congrès de l'AIRH - Séminaire $\mathrm{n}^{\circ} 4$ Cagliari, 10-14 Septembre 1979.

[2]BAUER, LARMINAUX, LEMOINE, SUREAU. - Simulation numérique des générateurs de vapeur nucléaires. $\mathrm{XII}^{\mathrm{e}}$ Journées de l'Hydraulique - Paris 1972.

[3] HENNEBICQ, TASSART. - PWR 900 - Etude de la régulation de niveau des générateurs de vapeur couplés entre eux $a$ a basse charge. Simulations analogique et numérique. Note Technique EDF-SEPTEN E-SE/FO 75-03 du 14 Avril 1975.

[4] HENNEBICQ, TASSART. - Tranches $W 900 \mathrm{MW}$ - Etude de la régulation de niveau des générateurs de vapeur. Proposition de modification du schema de régulation. Note technique EDF-SEPTEN, E-SE/FO 74-42 du 9 Juin 1975.

\section{Discussion}

Le Président. - Je remercie M. TASSART de sa communication. Y-a-t-il des questions à poser sur le problème des générateurs de vapeur?

M. MONDIN. - Vous avez fait allusion aux générateurs de vapeur Combustion Engineering et K.W.U. Y a-t-il des différences entre les G.V. construits en France et ces générateurs de vapeur?
M. TASSART. - La circulation y est naturelle, de la même façon. Il y a des différences au niveau du matériau : les tubes ne sont pas dans le même acier. Les plaques-supports ne sont pas de la même conception et les séparateurs cyclones sont eux aussi différents. Il n'y a pas d'autres différences fondamentales entre les GV de la K.W.U. et ceux qui sont construits en France. 
M. MONDIN. - N'y a-t-il pas de système économiseur ?

M. TASSART. - Il en existe sur les générateurs de vapeur qui vont bientôt démarrer, qu'ils soient de conception Westinghouse ou de conception K.W.U. En France, ce dispositif n'a pas encore été adopté car il ne semble pas garantir pour l'instant une fiabilité suffisante.
L'expérience de démarrage et de fonctionnement de ces tranches nous apportera certainement des enseignements intéressants tant au niveau de la connaissance du gain de pression effectif qu'apporte ce dispositif qu'au niveau de sa fiabilité après quelques années.

\section{Abstract \\ Two-phase problems in the steam generators of PWR boilers Transient low-pressure effects}

At rated operating conditions, emulsion passes through two-thirds of the secondary volume of the natural-circulation PWR steam-generators Framatome has produced for Electricite de France (EDF). The numerical data in Figure 1 are from an EDF-designed single-path numerical model.

\section{I - Tube corrosion}

Tube corrosion is accelerated in high heat-transfer flux areas in the vicinity of the tube plate by drying and re-wetting of the wall and sludge accumulation. Determined efforts have been made to obtain closer data on flow conditions at the foot of the tube cluster, by such means as eddy-current measurement, two-dimensional numerical two-phase models, comprehensive instrumentation of steam generators in operation, mock-ups, etc. The first three of these have successfully contributed to improvement of flow conditions at the foot of new French-designed steam generators.

Tube contraction and subsequent crack formation can also be caused by tube plate corrosion due to unsatisfactory 'sweeping' of the tubes at the plate joints. 'Quadrifoliate' design of tube passages through the plates is expected to improve steam generator reliability.

II - Possible hydroelastic instability of the tube cluster

Fluid/tube cluster interaction is significant at arch supports. Two unknowns for evaluating stability margins are 1) normal velocity distributions at the largest radii and 2) margins resulting from vapour/liquid slip.

\section{III - Performance}

The usefulness of a 'cold-leg' economizer mainly depends on the effect it will have on heat-transfer and corrosion conditions at the foot of both branches of the tube cluster. Closer data on the subject may be obtainable from operating experience with generators provided with an economizer and from tests on water-steam mock-ups.

Operating experience with steam generators and model tests have revealed the vital importance of the intermediate cyclone/drier area. By suitable layout design of this area it should be possible to ensure satisfactory steam quality from the generators with which EDF's $900 \mathrm{MWe}$ boilers are provided.

\section{IV - Hydrodynamic stability of the circulation loop}

Whereas stability of the $900 \mathrm{MWe}$ EDF steam generators has turned out to be adequate, the stability limits for the $1300 \mathrm{MWe}$ generators are a little off rated conditions. Closer knowledge of the head losses in the circulation loop is required for calculation of these limits. Tests on a model with freon should enable detailed investigation of the problem.

\section{V - Level measurement}

Steam generator level measurement by recording differential static pressure across two tappings on the same vertical (Fig. 1) fulfils both regulating and safety functions. Development work is in progress as regards the representativeness of this system in the case of accidental depressurization.

\section{VI - Level regulation at low load}

The level-regulating system provided on Westinghouse steam generators prior to the end of 1975 - manually-operated at below $15 \%$ load - was one of the main causes of unavailability during start-ups. This problem has been studied on a numerical axial two-phase steam generator simulation model designed by EDF.

Level response to a steam or water discharge increment (Figs. 4 and 5) takes place in two stages, 1) rapid redistribution of the mass of secondary water in the generator and 2) evidence of global mass balance variation showing up in the measured level. The greater 'settling' and 'swelling' amplitudes at low load are due to the non-linear relationship between depression and steam content.

The following improvements are based on operating experience, the numerical model and an analog model of two coupled steam generators (4):

a) A separate control system for the low-flow control valve (water and steam flow monitors are useless at below $15 \%$ load).

b) Dynamic adaptation of tendency action.

c) Dependence of proportional regulator gain on feed temperature.

d) Automatic switchover from 'low flow' to "high now' regulation.

This regulation system is a vast improvement over the manual system. Owing to the limited gain of the level regulator, however, not all pre-positioning deficiencies of the 'low flow'valve can be recovered.

\section{Conclusions}

Schematic single-path representation of natural-circulation steam generators has enabled development of a satisfactory level-regulating system.

Many different investigation methods have been applied in anti-corrosion research, as a result of which circulation rates have been increased and further problems have been solved or are expected to be so in the near future.

Further means of investigation and additional tests are being devised for operating security and cost-optimization research. 